\title{
Corrigendum: Potential Clinical Benefits of CBD-Rich Cannabis Extracts Over Purified CBD in Treatment-Resistant Epilepsy: Observational Data Meta-analysis
}

\author{
Fabricio A. Pamplona ${ }^{1 *}$, Lorenzo Rolim da Silva ${ }^{2}$ and Ana Carolina Coan ${ }^{3}$ \\ ${ }^{1}$ Entourage Phytolab, São Paulo, Brazil, ${ }^{2}$ Bedrocan Brasil, São Paulo, Brazil, ${ }^{3}$ UNICAMP, Campinas, Brazil
}

Keywords: cannabinoids, cannabidiol (CBD), epilepsy, meta-analysis, refractory epilepsy, phytotherapy

\section{A Corrigendum on}

Potential Clinical Benefits of CBD-Rich Cannabis Extracts Over Purified CBD in Treatment-Resistant Epilepsy: Observational Data Meta-analysis

by Pamplona, F. A., da Silva, L. R., and Coan, A. C. (2018). Front. Neurol. 9:759. doi: 10.3389/fneur. 2018.00759

In the original article, there was a mistake in Table 2 as published. The total number of one reference was wrongly included in the calculation of the endpoint efficacy over $70 \%$. Where it reads 83/430, it should be 83/311, therefore 27\% (instead of 19\%). The corrected Table 2 appears below.

In the original article, there was a mistake in Table 4 as published. Data from one reference

OPEN ACCESS

Edited and reviewed by: Richard Lowell Bell,

Indiana University, United States

*Correspondence:

Fabricio A. Pamplona fabriciopamplona@gmail.com

Specialty section:

This article was submitted to

Neuropharmacology,

a section of the journal

Frontiers in Neurology

Received: 02 November 2018 Accepted: 20 November 2018 Published: 10 January 2019

Citation:

Pamplona FA, da Silva LR and Coan AC (2019) Corrigendum: Potential Clinical Benefits of CBD-Rich Cannabis Extracts Over Purified CBD

in Treatment-Resistant Epilepsy:

Observational Data Meta-analysis.

Front. Neurol. 9:1050.

doi: 10.3389/fneur.2018.01050 was missing in the calculation of the endpoints mild AE and severe AE. Where it reads 285/663, it should be $308 / 663$, therefore $46 \%$ (instead of $43 \%$ ). Where it reads $64 / 487$, it should be $64 / 483$. The corrected Table 4 appears below.

In the original article, due to the errors in Tables 2, 4 mentioned above, corrections have been made to the Abstract as well as the Results, paragraphs one, two, three and five:

- " $81 / 223,36 \%$ " changed to " $81 / 175,46 \%$ " in Abstract and Results, paragraph one.

- " $p=0.56$ " changed to " $p=0.52$ " in Abstract and Results, paragraph two.

- “97/255, 38\%” changed to "122/330, 37\%” in Abstract and Results, paragraph two.

- "6.1 mg/kg/day" changed to " $6.0 \mathrm{mg} / \mathrm{kg} /$ day” in Abstract and Results, paragraph two.

- "27.1 mg/kg/day” changed to " $25.3 \mathrm{mg} / \mathrm{kg} /$ day” in Abstract and Results, paragraph two.

- "(109/285 vs. $291 / 346, p<0.0001)$ and severe (23/285 vs. $77 / 346, p<0.0001)$ " changed to "( $158 / 216,76 \%$ vs. $148 / 447,33 \%, p<0.001)$ and severe $(41 / 155,26 \%$ vs. $23 / 328,7 \%, p<0.0001)$ " in Abstract and Results, paragraph five.

- “17.7 mg/kg/day” changed to " $15.0 \mathrm{mg} / \mathrm{kg} /$ day” in Results, paragraph two.

- "18\%" changed to " $27 \%$ " in Results, paragraph three.

- " $83 / 430$ ” changed to " $83 / 311$ ” in Results, paragraph three.

- "(Table 4)" changed to reference "(11)" in Results, paragraph five.

The authors apologize for this error and state that this does not change the scientific conclusions of the article in any way. The original article has been updated.

Copyright $\odot 2019$ Pamplona, da Silva and Coan. This is an open-access article distributed under the terms of the Creative Commons Attribution License (CC BY). The use, distribution or reproduction in other forums is permitted, provided the original author(s) and the copyright owner(s) are credited and that the original publication in this journal is cited, in accordance with accepted academic practice. No use, distribution or reproduction is permitted which does not comply with these terms. 
TABLE 2 | Efficacy of treatments in the reduction of convulsive seizures (heterogeneous population).

\begin{tabular}{|c|c|c|c|c|c|}
\hline References & Patients & Reported improvement & $>50 \%$ & $>70 \%$ & Mean daily dose (mg/kg/day) \\
\hline CBD pure (6) & 137 & $37 \%$ & $37 \%$ & $22 \%$ & $22.9 \mathrm{mg} / \mathrm{kg}$ \\
\hline CBD pure (7) & 7 & $86 \%$ & $71 \%$ & $57 \%$ & $22 \mathrm{mg} / \mathrm{kg}$ \\
\hline CBD pure (10) & 48 & NR & $42 \%$ & NR & $28.2 \mathrm{mg} / \mathrm{kg}$ \\
\hline CBD-rich extract (11) & 19 & $84 \%$ & $74 \%$ & $42 \%$ & $7.0 \mathrm{mg} / \mathrm{kg}$ \\
\hline CBD-rich extract (12) & 117 & $85 \%$ & $N R$ & NR & $4.3 \mathrm{mg} / \mathrm{kg}$ \\
\hline CBD-rich extract (28) & 75 & $57 \%$ & $33 \%$ & $N R$ & NR \\
\hline CBD-rich extract (13) & 74 & $89 \%$ & $34 \%$ & $18 \%$ & $<10 \mathrm{mg} / \mathrm{kg}$ \\
\hline
\end{tabular}

Endpoints: any improvement reported, improvement > 50\% ("clinical responder") and >70\%, and average dose reported. NR, not reported; ?, inconclusive.

TABLE 4 | Negative secondary effects of treatment with CBD-rich Cannabis extracts and purified CBD described as secondary endpoints in the clinical studies.

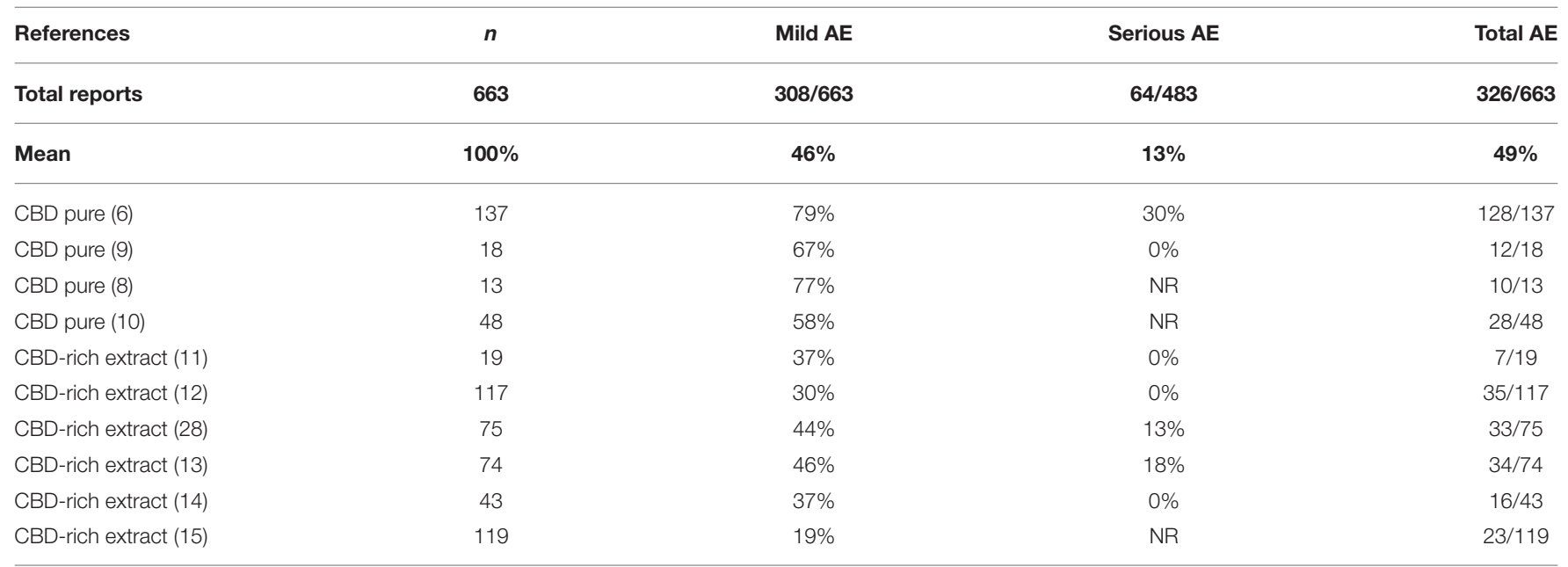

${ }^{*}$ Reporting adverse events in a study population does not necessarily mean that it is related to treatment. NR, not reported. 